\title{
Multi-band sub-GHz technology recognition on NVIDIA's Jetson Nano
}

\author{
Jaron Fontaine, Adnan Shahid, Robbe Elsas, Amina Seferagic, Ingrid Moerman and Eli De Poorter \\ IDLab, Department of Information Technology Ghent University - imec, Ghent, Belgium \\ jaron.fontaine@ugent.be
}

\begin{abstract}
Low power wide area networks support the success of long range Internet of things applications such as agriculture, security, smart cities and homes. This enormous popularity, however, breeds new challenging problems as the wireless spectrum gets saturated which increases the probability of collisions and performance degradation. To this end, smart spectrum decisions are needed and will be supported by wireless technology recognition to allow the networks to dynamically adapt to the ever changing environment where fair co-existence with other wireless technologies becomes essential. In contrast to existing research that assesses technology recognition using machine learning on powerful graphics processing units, this work aims to propose a deep learning solution using convolutional neural networks, cheap software defined radios and efficient embedded platforms such as NVIDIA's Jetson Nano. More specifically, this paper presents low complexity near-real time multi-band sub-GHz technology recognition and supports a wide variety of technologies using multiple settings. Results show accuracies around 99\%, which are comparable with state of the art solutions, while the classification time on a NVIDIA Jetson Nano remains small and offers real-time execution. These results will enable smart spectrum management without the need of expensive and high power consuming hardware.
\end{abstract}

Index Terms-Sub-GHz, deep learning, Software-defined radio, low-cost devices

\section{INTRODUCTION}

The Internet of things (IoT) paradigm has grown exponentially in the past decade and continues this trend into the foreseeable future. At the beginning of 2020, IoT Analytics estimated that 9.5 billion devices are connected to the Internet and forecasts a growth of 28 billion devices by 2025 [1]. This is due to the proliferation of various IoT application areas such as security, tracking, agriculture, smart metering, smart cities and smart homes. To accommodate commercial deployment of such large number of devices, recently a number of IoT technologies were developed which are called low power wide area networks (LPWANs). These technologies offer very long communication ranges allowing to connect a large number of devices using limited infrastructure cost (e.g. by installing a small number of gateways). Example technologies include Sigfox, LoRA, IEEE 802.11ah, IEEE 802.11g, Dash7, Weightless, etc. These technologies operate in unlicensed sub-GHz band, typically $868 \mathrm{MHz}$ in Europe and $915 \mathrm{MHz}$ in North America.

These radio frequencies offer good object penetration performance and can be used for a long range of communication, i.e., up to $15 \mathrm{~km}$ (for LoRa) [2]. However, due to

This work was funded by the Fund for Scientific Research Flanders (Belgium, FWO-Vlaanderen, FWO-SB grant number 1SB7619N). their low transmission speed and generally long on-air packet times, the risk of collisions between packet transmissions of multiple sensors is also increased, resulting in degraded overall performance. In Europe, these risks are mitigated by imposing duty cycle limitations (typically $1 \%$ per transmitter) [3] [4]. In addition, since LPWAN technologies operate in similar bands, it is quite possible that they interfere with each other, which further intensifies as the network density increases. As such, it becomes essential to identify co-existing technologies so that regulators can perform more accurate interventions and so that national and global LPWAN commercial network operators as well as local private deployments, e.g. in smart homes, can adapt the configurations of their networks to the local interference conditions (e.g. by switching to different radio frequencies, reducing the number of data transmissions, increasing the number of re-transmissions, etc.). Thus, it is imperative to have an identification solution that is flexible in supporting many existing as well as upcoming technologies and is characterized by low complexity and high generalization in various environments. Our proposed solution can be employed for enabling co-existence in such private LPWAN deployments.

To this end, our main contributions are as follows:

- We propose a multi-band deep learning (DL)-based technology recognition solution using convolutional neural network (CNN) capable of operating on multiple bands simultaneously.

- The solution is less complex and involves low-cost software-defined radios (SDRs) (SDR dongles) and efficient embedded platforms (NVIDIA Jetson Nano).

- The approach gives near real-time classification performance.

- In contrast to related work, we consider a variety of LPWAN technologies (Sigfox, LoRA, IEEE 802.15.4g and IEEE 802.11ah) and discuss their physical layer characteristics.

- We compare and analyze the performance of two different models (In-phase and quadrature components (IQ)based and fast Fourier transform (FFT)-based) in terms of accuracy, robustness against noise and classification processing speed.

The remainder of the paper is organized as follows. Section II presents related work on the topic of (sub-GHz) technology recognition. Section III presents the data capturing process 
using cheap SDR dongles and describes the data characteristics of the considered sub-GHz technologies. Next, in section IV we propose an efficient $\mathrm{CNN}$ for sub-GHz technology recognition, targeting the NVIDIA Jetson Nano embedded platform. The results are presented in section $\mathrm{V}$ and finally followed by conclusions in section VI.

\section{RELATED WORK}

Traditionally, identification of technologies is done by signal processing approaches such as energy detection, received signal strength indicator (RSSI)-based detection, cyclostationary feature detection [5], etc. However, such approaches cannot 1) work when multiple technologies coexist in similar bands and 2) scale to new technologies. During the past years, the world has seen tremendous improvements in DL, a subset of machine learning. Particularly, DL has dramatically improved stateof-the-art performance of various classification tasks such as speech recognition, object recognition, modulation classification and others. Most of the works on technology classification focus on $2.4 \mathrm{GHz}$ bands and the solutions developed there cannot be directly used for LPWAN technologies because of their peculiar characteristics such as narrow bandwidths, long packet duration and different modulation schemes.

In related work, the authors of [6] use deep neural network (DNN) and CNN for classification of Wi-Fi, DVB-T and LTE, and targets operation in multiple environments. The proposed models achieve an accuracy of $98 \%$, while still achieving $85 \%$ accuracy in low signal-to-noise ratio (SNR) scenarios $(0 \mathrm{~dB})$. The data which comprises of IQ samples was captured from a Universal Software Radio Peripheral (USRP) with a sampling rate of $10 \mathrm{Msps}$. The authors of [7] have successfully applied $\mathrm{CNN}$ to modulation recognition and achieve a similar accuracy of $85-90 \%$ in low SNR scenarios. Their dataset also comprises of IQ samples captured at a sampling rate of $1 \mathrm{msps}$. However, it contains simulated data with a proposal for capturing real data left as future work. Both approaches require expensive USRPs for data capturing and powerful graphics processing units (GPUs) for model training and validation. In contrast, this paper proposes an efficient model for classification of Sigfox, LoRA, IEEE 802.15.4g, and IEEE 802.11ah that is able to execute on a low-power embedded NVIDIA Jetson Nano GPU using cheap off the shelf SDR dongles. This work is the extension of our previous work on classification of LPWAN technologies (Sigfox, LoRA and IEEE 802.15.4g) [8], [9] where we used powerful GPUs for model training and validation. There are marked differences with our previous work: a) extension of LPWAN technologies by including IEEE 802.11ah and multiple settings for IEEE 802.15.4g, b) data capturing by low-cost off the shelf SDR dongles, and c) model design for training and validation on a low-power NVIDIA Jetson Nano GPU.

\section{SUB-GHz SIGNALS CAPTURED BY CHEAP SDR DONGLES}

In order to determine which technologies are transmitting, an appropriate method for sensing the wireless spectrum is

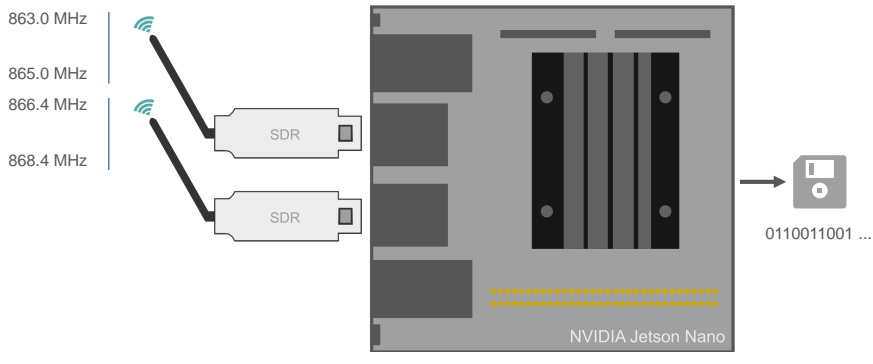

Fig. 1. Proposed framework of using SDR dongles with an NVIDIA Jetson Nano platform, capturing a wide band with minimal complexity and cost. For this paper we used two SDR dongles measuring two times $2 \mathrm{MHz}$ of bandwidth. This data is further processed by the NVIDIA Jetson Nano and saved for further analysis.

vital. For this, expensive hardware including SDR platforms exists such as USRPs that can easily capture wider bandwidth signals. For instance, USRP X310 can sample up to 200 Msps [10]. This, however, also requires expensive and energy consuming high-end hardware to process such a vast amount of data at a high speed. Moreover, LPWAN technologies do not operate on a very wide bandwidth. The technologies we focus on in this paper are LoRa, Sigfox, IEEE 802.11ah and IEEE $802.15 .4 \mathrm{~g}$. In Europe, these technologies operate around $868 \mathrm{MHz}$ and the precise operating center frequencies of the channels we have used from the technologies are given in Table I. In order to capture all the four technologies, it is required to capture the $5.4 \mathrm{MHz}$ band i.e., in the range from 863.0 $\mathrm{MHz}$ to 868.4 MHz. Unfortunately no cheap and energy constrained SDRs are available to capture such a $5.4 \mathrm{MHz}$ band.

\section{A. Sub-GHz capturing framework}

In this section we introduce a framework as shown in Figure 1 for cheap SDR dongles to capture IQ samples of a wide range of LPWAN technologies. Multiple SDR dongles are supported to simultaneously capture a wider band which is conventionally achieved through expensive and high performance USRPs. As such, we have used the RTL-SDR dongle capable of capturing up to $2.56 \mathrm{MHz}$ bandwidth [11]. By combining two SDR dongles we manage to capture IQ samples from a bandwidth of $4 \mathrm{MHz}$ as illustrated in Figure 1, where the first one captures a $2 \mathrm{MHz}$ band between $863 \mathrm{MHz}$ and $865 \mathrm{MHz}$ while the second one samples between $866.4 \mathrm{MHz}$ and $868.4 \mathrm{MHz}$. Note that we do not capture 1.4 MHz band from $865 \mathrm{MHz}$ to $866.4 \mathrm{MHz}$ due to the following reasons: a) in this band only IEEE 802.11ah partially exists, b) we want to classify all the four technologies from a setup with only two SDR dongles and those dongles cannot capture more than 4 $\mathrm{MHz}$ bandwidth without crashing and c) it is still possible to identify most of the IEEE 802.11ah signal variants, even with a partial signal. Next, the NVIDIA Jetson Nano stores the IQ samples of the two SDRs in memory for further processing. At the point of data collection, these IQ samples are written to a MATLAB compatible file. The files are pre-processed for the training phase (see Section IV) as follows: 


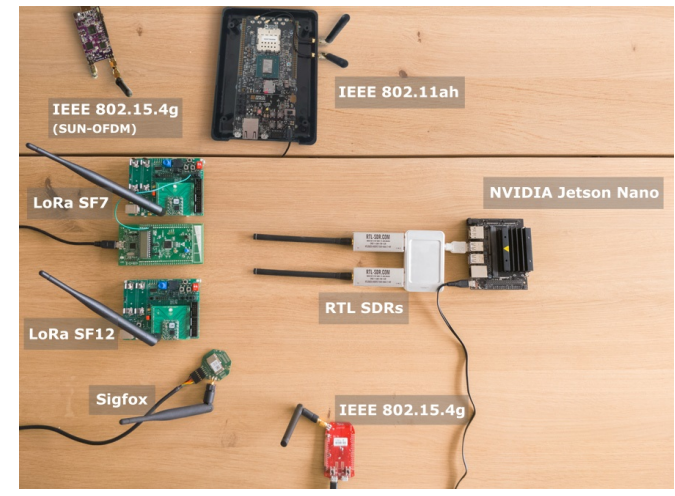

Fig. 2. Representation of system setup for data capturing. One Jetson captures the wireless spectrum, with the support of multiple SDR RTL dongles. The bottom half of the picture contains technologies captured in the $867 \mathrm{MHz}$ band (IEEE 802.15.4g SUN-FSK, Sigfox, LoRa SF7 and SF12, while the top half contains technologies that are mostly in the $864 \mathrm{MHz}$ band (IEEE 802.15.4g SUN-OFDM and IEEE 802.11ah).

1) The technology in the data collection experiment is noted a ground truth label.

2) The FFT of the IQ stream is optionally calculated.

3) Empty signals, where only noise is present, are removed from the training dataset.

4) The IQ or FFT data stream are divided into frames of 500. These frames are the input vectors provided to the model for classification. Each frame additionally contains the technology label.

5) The data is exported in a format compatible with the TensorFlow machine learning platform.

\section{B. Sub-GHz technology characteristics}

Figure 2 shows a representation of the setup we used for data capturing ${ }^{1}$. In total, the IQ samples of four technologies are captured. Moreover, the technologies have different physical layer settings such as center frequency, bandwidth, and modulation and coding scheme (MCS).

Table I provides an overview of the settings for each technology that we have used.

- The two LoRa signal classes operate both at $868.1 \mathrm{MHz}$, with a bandwidth of $125 \mathrm{KHz}$ and use a proprietary spread spectrum modulation. The difference between LoRa with spreading factor (SF) 7 and 12 is the packet duration (0.0698 vs 1.715 seconds) and symbol duration (1.02 vs 32.77 milliseconds) [12].

- Our dataset additionally contains Sigfox signals that use binary phase-shift keying (BPSK) modulation and are centered at a frequency of $868.2 \mathrm{MHz}$. The signal is characterized by 400 different orthogonal channels with each of bandwidth $100 \mathrm{~Hz}$.

- The dataset also includes IEEE 802.11ah (also known as Wi-Fi HaLow). This signal class was captured at four different channels, two different bandwidths and three

\footnotetext{
${ }^{1}$ The dataset is accessible here: https://github.com/ewine-project/SubGHztechnologies-dataset-Sigfox-LoRA-and-IEEE802.15.4g-subGHz-
}

TABLE I

THE VARIETY OF THE DATASET CAN BE NOTED WITH THE SETTINGS OF THE USED TECHNOLOGIES. THESE TECHNOLOGIES INCLUDE LORA WITH SPREADING FACTOR SF 7 AND SF 12, SIGFOX, IEEE 802.11 AH (WI-FI

HALOW) AND IEEE 802.15.4G (SUN-FSK AND SUN-OFDM).

\begin{tabular}{|c|c|c|c|}
\hline Technology & Center frequency & Bandwidth & Modulation \\
\hline LoRa SF 7 & $868.1 \mathrm{MHz}$ & $125 \mathrm{KHz}$ & Spread spectrum \\
\hline LoRa SF 12 & $868.1 \mathrm{MHz}$ & $125 \mathrm{KHz}$ & Spread spectrum \\
\hline Sigfox & $868.2 \mathrm{MHz}$ & $100 \mathrm{~Hz}$ & BPSK (400 chan.) \\
\hline \multirow[t]{4}{*}{ IEEE 802.11ah } & 863.5 MHz & $1 \mathrm{MHz}$ & $\begin{array}{r}\text { MCS } 0,10 \text { (BPSK), } \\
7 \text { (64-QAM) }\end{array}$ \\
\hline & $864.0 \mathrm{MHz}$ & $2 \mathrm{MHz}$ & $\begin{array}{r}\text { MCS } 0 \text { (BPSK), } \\
7 \text { (64-QAM) }\end{array}$ \\
\hline & $864.5 \mathrm{MHz}$ & $1 \mathrm{MHz}$ & $\begin{array}{r}\text { MCS } 0,10 \text { (BPSK), } \\
7 \text { (64-QAM) }\end{array}$ \\
\hline & $866.0 \mathrm{MHz}$ & $2 \mathrm{MHz}$ & $\begin{array}{r}\text { MCS } 0 \text { (BPSK), } \\
7 \text { (64-QAM) }\end{array}$ \\
\hline $\begin{array}{l}\text { IEEE } 802.15 .4 \\
\text { SUN-FSK }\end{array}$ & $868.1 \mathrm{MHz}$ & $200 \mathrm{KHz}$ & BFSK \\
\hline \multirow{5}{*}{$\begin{array}{l}\text { IEEE } 802.15 .4 \\
\text { SUN-OFDM }\end{array}$} & $863.625 \mathrm{MHz}$ & $1.2 \mathrm{MHz}$ & MCS 2 (OQPSK) \\
\hline & $863.425 \mathrm{MHz}$ & $800 \mathrm{KHz}$ & MCS 2 (OQPSK) \\
\hline & $863.225 \mathrm{MHz}$ & $400 \mathrm{KHz}$ & MCS 2 (OQPSK) \\
\hline & $863.125 \mathrm{MHz}$ & $200 \mathrm{KHz}$ & MCS 2 (OQPSK) \\
\hline & $863.125 \mathrm{MHz}$ & $200 \mathrm{KHz}$ & MCS 6 (16-QAM) \\
\hline
\end{tabular}

different MCSs, with two types of modulations, BPSK and 64-quadrature amplitude modulation (QAM). HaLow operates at the highest bandwidth among the considered technologies and is capable of achieving the highest data rate (up to 78 Mbps) [13] [14].

- The signal class IEEE $802.15 .4 \mathrm{~g}$ comprises of three modulation schemes such as single carrier binary frequencyshift keying (BFSK) and multiple carrier offset quadrature phase-shift keying (OQPSK) and 16-QAM. This class can further be divided into two sub classes. The first one is called SUN-FSK while the second one is the SUN-OFDM physical layer [14]. We collected IQ samples from 5 variants of SUN-OFDM with different center frequencies, bandwidths, and modulations.

Figures 3 and 4 present the physical layer (PHY) characteristics of the signals using spectrograms generated from the dataset. These figures illustrate different packets intervals, packet lengths and bandwidths. With the addition of many signals using different modulation schemes, diverse features exist in the signal for technology recognition to correctly identify the signal classes. It should be noted that the spectrograms were not used for (automatic) feature extraction by the CNN presented in IV, instead the model uses independent time domain or frequency domain features.

\section{EFFICIENT CNN FOR SUB-GHZ TECHNOLOGY RECOGNITION}

Wireless technology recognition promises great potential due to accurate classification with state-of-the-art (SOTA) machine learning techniques. The literature already describes numerous of supervised learning models such as DNNs and CNNs for a vast range of applications i.e. image recognition, optical character recognition, spam detection, pattern recognition, etc. For wireless technology recognition, most models 

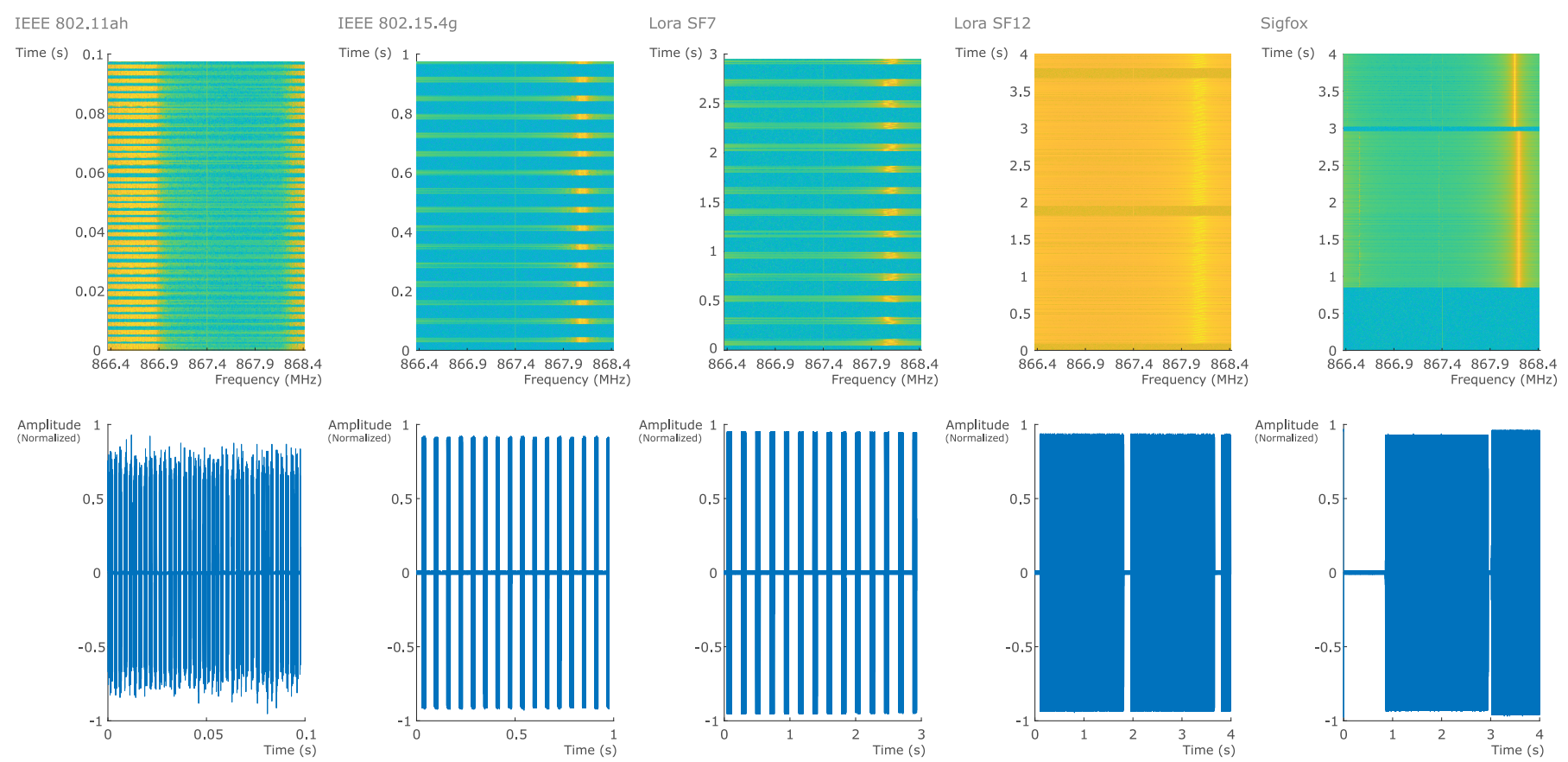

Fig. 3. Spectrogram and amplitude plots of IEEE 802.11ah, IEEE 802.15.4g, Lora with spreading factor 7, Lora with spreading factor 12 and Sigfox in the 867.4 $\mathrm{MHz}$ band showing the differences in packet length, sending interval and bandwidth.
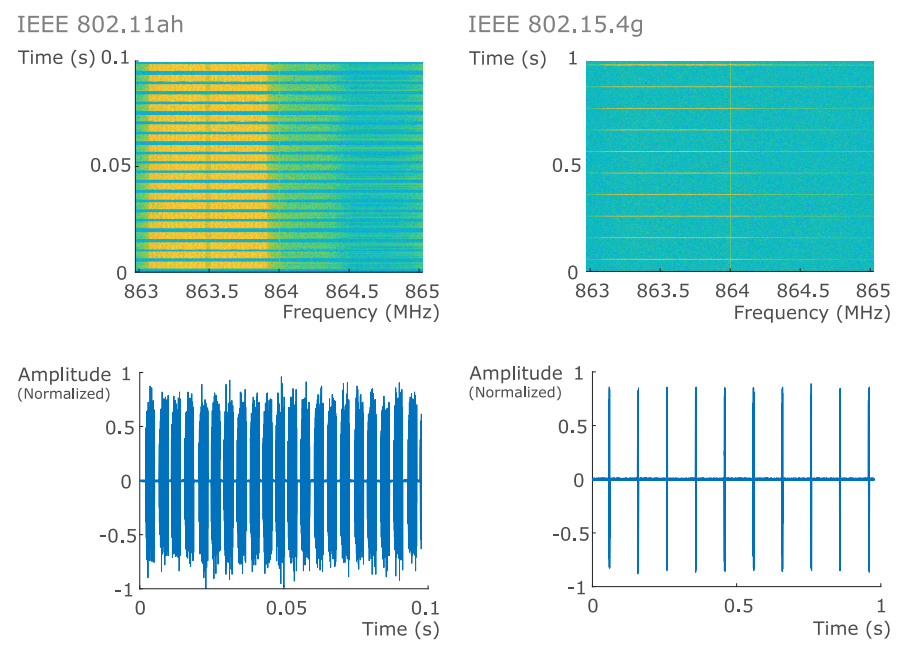

Fig. 4. Spectrogram and amplitude plots of IEEE 802.11ah and IEEE 802.15.4g in the $864 \mathrm{MHz}$ band showing the differences in packet length, sending interval and bandwidth.

are designed to run on high-end hardware such as servers, laptops and desktops with expensive GPUs. To bring technology recognition closer to embedded hardware platforms, we designed a CNN capable of running near real-time on embedded GPUs e.g. NVIDIA Jetson Nano.

\section{A. Input sample description}

In total, four models are trained and evaluated. Two model variations exist because of the focus on two different channels (864 MHz and 867.4 MHz). Additionally, two types of input
TABLE II

CONVOLUTIONAL NEURAL NETWORK ARCHITECTURE

\begin{tabular}{ll}
\hline Layer & Output dimension \\
\hline Input & $500 \times 2 \times 1$ \\
Conv $(8,2 \times 1)$, relu & $500 \times 2 \times 8$ \\
Max pool $(2 \times 1)$ & $250 \times 2 \times 8$ \\
Batch normalization & $250 \times 2 \times 8$ \\
Dropout $10 \%$ & $250 \times 2 \times 8$ \\
Conv $(4,3 \times 2)$, relu & $250 \times 2 \times 4$ \\
Max pool $(4,2)$ & $62 \times 1 \times 4$ \\
Dropout $10 \%$ & $62 \times 1 \times 4$ \\
Batch normalization & $62 \times 1 \times 4$ \\
Flatten & 248 \\
Dense $(50)$, relu & 50 \\
Dropout 25\% & 50 \\
Dense (16), relu & 16 \\
Output $(6$ or 3), softmax & 6 or 3
\end{tabular}

data are considered: a) raw IQ samples and b) FFT of the IQ samples. In both types, each input example to the model is of dimension $500 \mathrm{X} 2$. For the IQ case, the first 500 values correspond to I components while the second 500 values represent $\mathrm{Q}$ components. For the FFT case, the IQ data is transformed into its equivalent real and imaginary frequency domain representation using FFT. This transformation produces a more robust model especially in low SNR environments [6].

\section{B. Model architecture}

Table II presents the novel embedded-focused CNN model architecture. The first layers extract features from the signals 
TABLE III

COMPLEXITY ANALYSIS: MODEL PARAMETERS AND MACS

\begin{tabular}{lll} 
Model & Parameters & MACs \\
\hline 867 MHz model & 13,636 parameters & 112,000 MACs \\
864 MHz model & 13,858 parameters & 112,000 MACs \\
Model [9] & 8,008,545 parameters & $3,456,000$ MACs \\
Model [8] & 1,023,122 parameters & $7,104,000$ MACs
\end{tabular}

using convolutional layers. The Max pool layers effectively reduce the dimensionality, with the aim to lower the complexity of the model. That is, by reducing the amount of fully connected neurons before the dense layers, we can greatly minimize the amount of trainable parameters. Moreover, from our experience, batch normalization improves the training speed and stability by normalizing the output of the activation functions of the previous layer. Dropout further prevents the model to overfit, which can otherwise lead to models that cannot generalize towards unseen data. The fully connected layers are kept small to further minimize the model footprint. Finally, the output uses a softmax activation function to learn to differentiate 6 signal classes (802.11ah, 802.15.4g, LoRa SF7, LoRa SF12, SigFox and noise in the 867.4 MHz band) or 3 signal classes $(802.11 \mathrm{ah}, 802.15 .4 \mathrm{~g}$ and noise in the 864 $\mathrm{MHz}$ band). The softmax function is defined as follows (1):

$$
\operatorname{softmax}(z)_{i}=\frac{e^{z_{i}}}{\sum_{j} e^{z_{j}}} .
$$

where $j=1, \ldots, N$ and $z_{i}$ is

$$
z_{i}=\sum_{k} p_{k} W_{k i} .
$$

where $N$ is the number of signal classes, $i$ is the considered output neuron, $k=1, \ldots$, SizePreviousLayer, $p_{k}$ is the output of the previous layer's neuron and $W_{k i}$ is the weight of the $i$ th neuron learned at $p_{k}$.

The complexity analysis in terms of model parameters and MACs is presented in Table III. Compared to the related work, this model offers 75 to 580 times less parameters. Another way to specify the model's computational footprint is to look at the amount of multiply-accumulate (MAC) operations in the convolutional layers [15]. Moreover, a linear relationship between the MAC count and energy consumption of CNN models exists which can help to compare models suitable for embedded devices [16]. For each convolutional layer, the amount of MAC operations is given in (3):

$$
M A C_{l}=F_{l, x} \times F_{l, y} \times F_{l, n} \times I_{l-1, x} \times I_{l-1, y} \times I_{l-1, z} .
$$

where $F_{l, x}$ and $F_{l, y}$ are the 2D filter dimensions, $x$ and $y$ represent Cartesian axes, $F_{l, n}$ is the number of filters of the $l$ th layer, and $I_{l-1, x}, I_{l-1, y}, I_{l-1, z}$ are the input dimensions from the previous $l-1$ th layer.

Again, a clear difference in computational complexity is visible as the proposed model contains 30 to 63 times less MACs and is thus more appropriate for embedded devices.

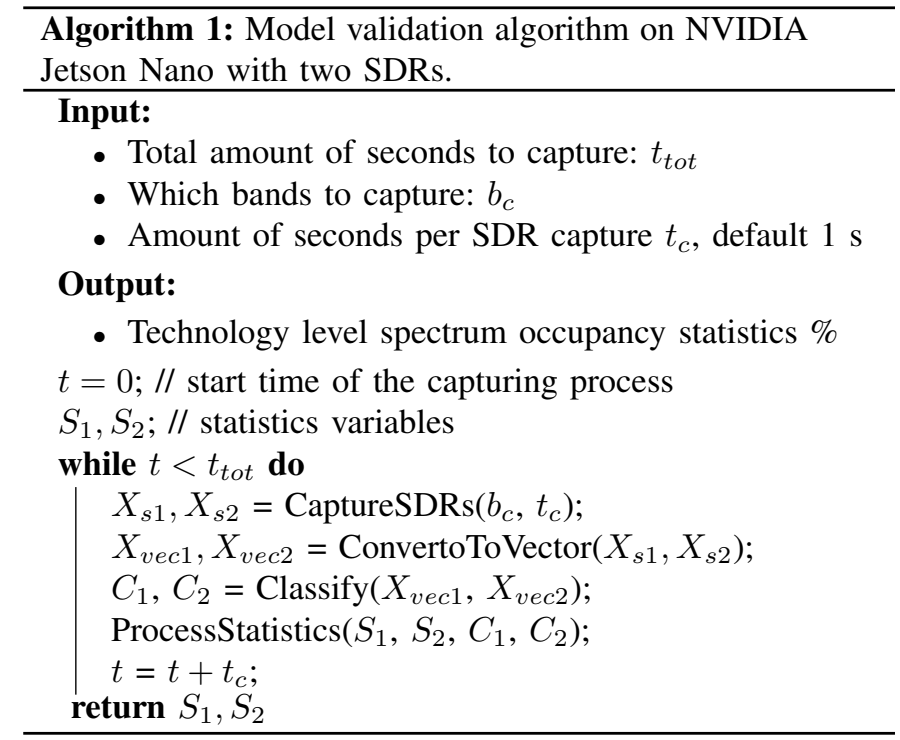

\section{Model implementation}

The model was trained using the Adam optimizer with a learning rate of 0.001 and a batch size of 64. After we captured the data, as described in III-A, an offline learning process took place on a desktop computer with NVIDIA RTX TITAN graphics card and an Intel ${ }^{\circledR}$ Core ${ }^{\text {TM }}$ CPU i9-9900K @ 3.60GHz. Tensorflow and Keras are two Python libraries that allowed us to train the model in a straightforward fashion. The model was relocated to the NVIDIA Jetson Nano for validation after the training process. Tensorflow and Keras are also compiled onto the NVIDIA Jetson Nano and employ the model with the embedded dedicated 128-core Maxwell GPU.

\section{Validation algorithm}

Once the trained models are transferred, the NVIDIA Jetson Nano establishes the validation process . Algorithm 1 describes the flow carried out from capturing the signals of two sub-GHz bands using two SDR dongles to classifying the associated technologies with the models. The algorithm expects the amount of seconds $\left(t_{c}\right)$ and which bands to capture $\left(b_{c}\right)$. For the amount of time $t_{c}$, new samples $X_{s}$ are captured for all SDRs simultaneously. By default, the SDRs capture for 1 second in each iteration. Next, before the signal classifier predicts the signal classes $C$, input data is split into compatible input vectors $X_{v e c}$. The signal classes are processed into statistics $S$ before the loop continues until $t_{c}$ is satisfied. Finally, the output of the algorithms returns spectrum occupancy statistics. More specifically, for each technology, on each band, occupancy percentages are calculated.

\section{Results}

This section describes the results of the two models (IQbased and FFT-based) on two different channels in terms of a) model accuracy, b) robustness to noise and c) model processing speed. First the classification accuracy, precision and recall of the models are presented. Results are achieved 
TABLE IV

MOdEL ACCURACY, PRECISION, RECALL AND F1 SCORE

\begin{tabular}{lllll} 
Model & Accuracy & Precision & Recall & F1 score \\
\hline 867 MHz IQ & $98.89 \%$ & $98.90 \%$ & $98.89 \%$ & $98.89 \%$ \\
867 MHz FFT & $98.33 \%$ & $98.37 \%$ & $98.33 \%$ & $98.33 \%$ \\
864 MHz IQ & $99.80 \%$ & $99.80 \%$ & $99.80 \%$ & $99.80 \%$ \\
864 MHz FFT & $99.92 \%$ & $99.92 \%$ & $99.92 \%$ & $99.92 \%$
\end{tabular}

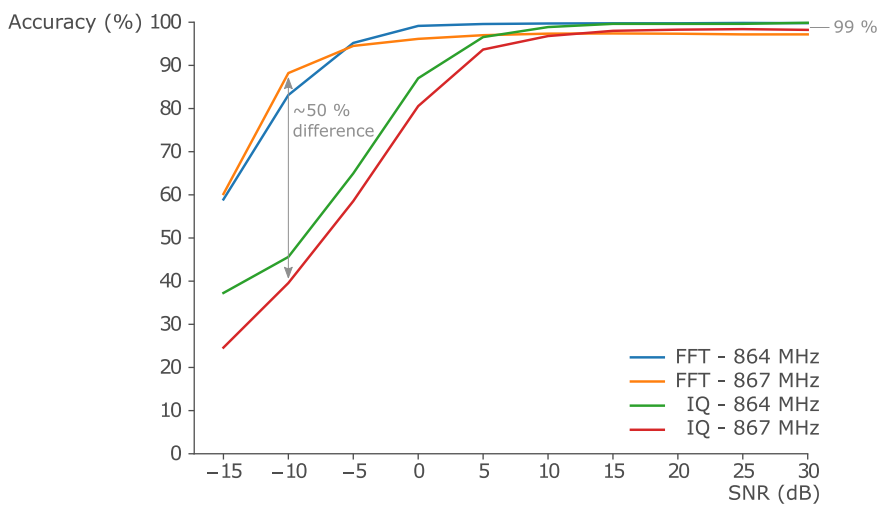

Fig. 5. SNR plot comparing the higher accuracy of FFT mode models to the lower accuracy of IQ mode models in low SNR scenarios (up to approximately $50 \%$ difference). This gap is diminished in high SNR scenarios (around 99 $\%$ accuracy).

by shuffling the datasets and taking a random split of $60 \%$ / $40 \%$ for training and validation data, respectively. Next, various levels of noise (10 SNR levels i.e., $-15,-10, \ldots$, $30 \mathrm{~dB}$ ) are introduced into the dataset which allows to asses the model's effectiveness in environments with different SNR conditions. Finally we experimentally measure the processing and classification speed on the NVIDIA Jetson Nano to analyze the processing performance of the proposed model.

\section{A. Model accuracy}

Table IV presents the accuracy of the four trained models together with the average precision and recall. The accuracy of the $864 \mathrm{MHz}$ model (>99.8\%) is slightly higher in both FFT and IQ mode compared to the $867 \mathrm{MHz}$ model (>98.3\%). We believe this can be explained by the model having less signal classes to identify. Furthermore, the two signals in the $864 \mathrm{MHz}$ band, 802.11ah and 802.15.4g SUN-OFDM, have clearly distinct features such as different modulation schemes and bandwidths. The difference between IQ and FFT mode is less pronounced. The signals in the $867 \mathrm{MHz}$ band achieve a slightly higher accuracy in the IQ mode (98.9\% vs $98.33 \%$ ), whereas the opposite is true for signals in the $864 \mathrm{MHz}(99.8 \%$ vs $99.9 \%$ ). We can conclude that time domain features are sufficient in most scenarios where the signal is clear and high above the noise floor. Finally, compared to related research [9] [8], the model retains and even surpasses classification accuracy (98.9\% and $99.8 \%$ compared to $95 \%$ and $97 \%$ in IQ mode), even with reduced complexity for embedded platforms.
TABLE $\mathrm{V}$

PROCESSING AND CLASSIFICATION TIME ON NVIDIA's JETSON NANO

\begin{tabular}{llll} 
Model & $\begin{array}{l}\text { Pre-processing } \\
\text { time }\end{array}$ & $\begin{array}{l}\text { Classification } \\
\text { time }\end{array}$ & Total time \\
\hline IQ model & $182 \mathrm{~ms} \pm 3 \mathrm{~ms}$ & $769 \mathrm{~ms} \pm 7 \mathrm{~ms}$ & $951 \mathrm{~ms}$ \\
FFT model & $311 \mathrm{~ms} \pm 2 \mathrm{~ms}$ & $847 \mathrm{~ms} \pm 7 \mathrm{~ms}$ & $1158 \mathrm{~ms}$
\end{tabular}

\section{B. Robustness to noise}

The models' robustness to noise is presented in Figure 5. A big gap between the accuracy of the IQ and FFT mode is noticeable in the low SNR levels between -15 and $0 \mathrm{~dB}$. This gap reaches a maximum difference of 50\% accuracy around a SNR of $-10 \mathrm{~dB}$. Signals in the frequency domain are generally less affected by noise, because noise should not have any fixed recurring frequency component. As such, the frequency components of the signal stand out whereas signals in time domain are masked by the noise floor. This causes the model to recognize features with a higher confidence in the FFT mode compared to the IQ mode. Again, compared to other related research [9] [8] the proposed small CNN achieves comparable performance, if not higher, caused by optimizations to the model. Similar to the conclusion in section V-A, we observe a higher accuracy for high SNR scenarios $(>10 \mathrm{~dB})$ in IQ mode for the $867 \mathrm{MHz}$ signals and a negligible difference for the $864 \mathrm{MHz}$ signals. Overall, the accuracies for the upper SNR levels settle around 99\%.

\section{Processing and classification time}

To determine the feasibility of sub-GHz technology recognition on an embedded platform such as the NVIDIA Jetson Nano, we measured the processing and classification time on the platform according to the algorithm described in section IV-D. Table $\mathrm{V}$ highlights the average (pre)processing time and classification time for each model on the NVIDIA Jetson Nano. Each measurement of 1 second of wireless signals takes on average (100 runs) in total $951 \mathrm{~ms}$ for the IQ model. This means that the total processing speed for that model is faster than the capturing duration and can enable near-real time classification (assuming the SDRs continue to capture during the processing step). For the FFT model, this duration increases to $1158 \mathrm{~ms}$ because of the FFT function that the pre-processing step executes. Still, for a very robust model, near-real time classification is feasible.

\section{CONCLUSION}

The rapid success of LPWAN IoT technologies raises challenging problems such as smart spectrum management. Wireless technology recognition in sub-GHz bands can benefit the performance of many such wireless IoT applications. In contrast to state-of-the-art solutions, this work presents a low cost CNN solution using cheap SDR devices and can scale towards multiple bands, covering most of Europe's $868 \mathrm{MHz}$ bands. The proposition can also be applied to North America's $915 \mathrm{MHz}$ band. Validation of the proposed efficient models on NVIDIA's Jetson Nano embedded platform demonstrates 
near real-time classification capabilities. Results show high accuracies around $99 \%$ for both FFT and IQ models, while the FFT model still performs well in low SNR scenarios with $50 \%$ higher accuracy compared to it's IQ counterpart. A processing and classification time of $951 \mathrm{~ms}$ and $1158 \mathrm{~ms}$ is achieved for the IQ and FFT models respectively, enabling near-real time classification. Conclusively, the outcome of this paper can drastically impact the feasibility of intelligent spectrum management for low cost solutions.

\section{REFERENCES}

[1] K. L. Lueth, "Iot 2019 in review: The 10 most relevant iot developments of the year," January 2020. [Online]. Available: https://iot-analytics.com/iot-2019-in-review/

[2] V. Stoynov, V. Poulkov, and Z. Valkova-Jarvis, "Low power wide area networks operating in the ism band- overview and unresolved challenges," in Future Access Enablers for Ubiquitous and Intelligent Infrastructures, V. Poulkov, Ed. Cham: Springer International Publishing, 2019, pp. 96-109.

[3] European Electronic Communications Committee, "ERC Recommendation 70-03," June 2019.

[4] The european commission, "Decision 2006/771/ec on harmonisation of the radio spectrum for use by short-range devices and repealing decision 2006/804/ec."

[5] K. Kim, I. A. Akbar, K. K. Bae, J.-S. Um, C. M. Spooner, and J. H. Reed, "Cyclostationary approaches to signal detection and classification in cognitive radio," in 2007 2nd IEEE International Symposium on New Frontiers in Dynamic Spectrum Access Networks. IEEE, 2007, pp. $212-215$.

[6] J. Fontaine, E. Fonseca, A. Shahid, M. Kist, L. A. DaSilva], I. Moerman, and E. D. Poorter], "Towards low-complexity wireless technology classification across multiple environments," Ad Hoc Networks, vol. 91, p. 101881, 2019. [Online]. Available: http://www.sciencedirect.com/science/article/pii/S1570870518309685

[7] T. J. O'Shea, J. Corgan, and T. C. Clancy, "Convolutional radio modulation recognition networks," in Engineering Applications of Neural Networks, C. Jayne and L. Iliadis, Eds. Cham: Springer International Publishing, 2016, pp. 213-226.

[8] A. Shahid, J. Fontaine, M. Camelo, J. Haxhibeqiri, M. Saelens, Z. Khan, I. Moerman, and E. De Poorter, "A convolutional neural network approach for classification of lpwan technologies: Sigfox, lora and ieee 802.15. 4g," in 2019 16th Annual IEEE International Conference on Sensing, Communication, and Networking (SECON). IEEE, 2019, pp. $1-8$.

[9] A. Shahid, J. Fontaine, J. Haxhibeqiri, M. Saelens, Z. Khan, I. Moerman, and E. De Poorter, "Demo abstract: Identification of lpwan technologies using convolutional neural networks," in IEEE INFOCOM 2019-IEEE Conference on Computer Communications Workshops (INFOCOM WKSHPS). IEEE, 2019, pp. 991-992.

[10] X300/X310, “Usrp x300/x310." [Online]. Available: https://kb.ettus.com/X300/X310

[11] RTL-SDR, “About RTL-SDR," June 2020. [Online]. Available: https://www.rtl-sdr.com/about-rtl-sdr/

[12] T. L. Alliance, "What are LoRa $®$ and LoRaWAN@?" June 2020. [Online]. Available: https://lora-developers.semtech.com/library/techpapers-and-guides/lora-and-lorawan/

[13] E. De Poorter, J. Hoebeke, M. Strobbe, I. Moerman, S. Latré, M. Weyn, B. Lannoo, and J. Famaey, "Sub-ghz lpwan network coexistence, management and virtualization: an overview and open research challenges," Wireless Personal Communications, vol. 95, no. 1, pp. 187-213, 2017.

[14] H. Nguyen, N.-T. Le, N. Hoan, and Y. M. Jang, "Real-time mitigation of the mobility effect for ieee $802.15 .4 \mathrm{~g}$ sun mr-ofdm," Applied Sciences, vol. 9, p. 12, 082019.

[15] V. Akhlaghi, A. Yazdanbakhsh, K. Samadi, R. K. Gupta, and H. Esmaeilzadeh, "Snapea: Predictive early activation for reducing computation in deep convolutional neural networks," in 2018 ACM/IEEE 45th Annual International Symposium on Computer Architecture (ISCA), 2018, pp. 662-673.
[16] C. F. Rodrigues, G. Riley, and M. Luján, "Synergy: An energy measurement and prediction framework for convolutional neural networks on jetson tx1," in Proceedings of the International Conference on Parallel and Distributed Processing Techniques and Applications (PDPTA). The Steering Committee of The World Congress in Computer Science, Computer ..., 2018, pp. 375-382. 